\title{
Novel miRNA Sponges to Specifically Modulate Gene Expression in Colon Cancer Cells
}

\author{
Alejandro Aguilera ${ }^{1}$, Gloria Perazzoli ${ }^{1,3}$, Laura Cabeza $^{1,2}$, Cristina Mesas $^{1,2}$, Francisco \\ Quiñonero ${ }^{1,2}$, \\ Beatriz García-Pinel ${ }^{1,2}$, Celia Vélez ${ }^{1,2,3}$, Ana R. Rama ${ }^{1,2}$,
}

\author{
${ }^{1}$ Institute of Biopathology and Regenerative Medicine (IBIMER), Center of Biomedical Research \\ (CIBM), University of Granada, Granada, Spain. \\ ${ }^{2}$ Department of Anatomy and Embryology, Faculty of Medicine, University of Granada, Granada, Spain. \\ ${ }^{3}$ Biosanitary Institute of Granada (ibs. GRANADA), SAS-Universidad de Granada, Granada, Spain.
}

Correspondence: mariaceliavelez@ugr.es

\begin{abstract}
RNA sponges allow the selective blockade of a complete family of associated miRNAs which induce posttranscriptional gene silencing in its target through binding to 3'UTR mRNA. MiRNA-365 and miRNA-145 are down-regulated in colorectal cancer (CRC), but not in health tissues. Based on this, we constructed two vectors by inserting miRNA sponge (one for miRNA-365 and other for miRNA-145), and used EGFP (enhanced green fluorescent protein) as a $3^{\prime}$ UTR reporter gene to analyse the ability of each sponge to catch its respective miRNA. qPCR results corroborated that the expression levels of both miRNAs were lower in CRC cell lines than in normal colon cell line. Flow cytometry analysis revealed a decrease of the EGFP expression levels in the cell lines transfected with both sponges, being higher on the normal cell line while CRC cell lines presented a minimal decline. Also, this decrease was inversely proportional to the levels of expression of both miRNAs obtained by qPCR. These results were corroborated by fluorescence microscopy, showing a similar decrease fluorescence. We propose a new vector system to carry in a specific way the expression of genes to CRC cells without affecting healthy cells, preventing damage to healthy tissues.
\end{abstract}

Keywords: miRNA, sponges, miRNA-365; miRNA-145; CRC 


\section{Introduction}

Colorectal cancer (CRC) is the third most commonly occurring cancer in men and the second most commonly occurring cancer in women, and the second in terms of mortality [1]. The risks of metastasis are very high, being this the main cause of mortality. The five-year survival in CRC patients diminishes from $90 \%$, if the cancer is localized, to $68 \%$ if it has spread to contiguous organs and/or to lymphatic nodes [2]. This aggressiveness and the need of more effective treatments have led to the study of the miRNAs, which are found suppressed or overexpressed in this type of cancer [3].

MicroRNAs (miRNAs) are short non-coding RNAs, of $\sim 22$ nucleotides, which induce posttranscriptional gene silencing in its target through binding to 3'UTR mRNA [4]. The miRNAs are involved in diverse biological processes such as cell cycle control [5], cellular differentiation [6], development [7] and metabolism [8]. They are also involved in diseases such as diabetes [9], neurodegenerative diseases [10] and cancer [11,12]. Around 50\% of the miRNAs are located in 'fragile' areas of the genome, regions of loss of heterozygosity or breakpoints associated with cancer [13].

The expression profiles of the miRNAs are characterised by differing between normal and tumoural tissues, as well as among types of tumours [14]. These characteristic profiles can be used as biomarkers for the determination of prognosis, response to chemotherapy, prediction of the efficiency of the treatment and susceptibility of a patient to suffer a disease [3] (Table 1).

\begin{tabular}{|l|l|l|}
\hline miRNA & Function & Reference \\
\hline miRNA-129 & Induces cell apoptosis & {$[15]$} \\
\hline $\begin{array}{l}\text { miRNA- 200; miRNA-143/miRNA- } \\
145\end{array}$ & Tumour suppressor & {$[16-18]$} \\
\hline miRNA-16-1 & Cell cycle and apoptosis regulation & {$[19]$} \\
\hline miRNA- 126 & Biomarker for treatment response & {$[13]$} \\
\hline miRNA-145; miRNA-190 & Biomarker for early detection & {$[20][21]$} \\
\hline miRNA-150 & Tumour stage biomarker & {$[22]$} \\
\hline $\begin{array}{l}\text { miRNA-532-3p, }-331,-195,-17,- \\
142-3 p,-15 b,-532 \text { and }-562\end{array}$ & Identify quickly polyps & {$[23]$} \\
\hline miRNA-431, -15b and $-139-3 p$ & Stage IV CRC & {$[23]$} \\
\hline $\begin{array}{l}\text { miRNA-9, }-29 b,-127-5 p,-138,- \\
143,-146 a,-222, \text { and }-938,\end{array}$ & Disease progression & {$[24]$} \\
\hline miRNA-365 & & {$[25,26]$} \\
\hline miRNA.211-3p & Regulate cell proliferation & {$[27]$} \\
\hline
\end{tabular}

Table 1: MiRNA involved in CRC

The expression of the miRNA-129 induces cell apoptosis through the cell cycle arrest between phases G1 and G2. This miRNA is found deregulated in patients with CRC in stage II and IV [15]. The miRNA-200 family act as tumour suppressors in the epithelial-mesenchymal transition, being the mirRNA-200b related to cell growth, and its down-regulation to a worse 
prognosis and metastasis in CRC[28]. The miRNA-16-1 participates in processes of regulation of apoptosis and cell cycle. Its negative regulation of the KRAS expression entails the inhibition of tumour growth in CRC, thus being a great candidate to becoming into a therapeutic target [19]. MiRNA -126, whose expression seems to be bound to the expression of VEGF-A ( vascular endothelial growth factor A), could be used as response biomarker to a certain treatment [13]. Also, miRNA plasma panels (mir-532-3p, -331, -195, -17, -142-3p, -15b, -532 and -562) can be used to quickly identify the polyps in plasma or (mir-431, $-15 \mathrm{~b}$ and $-139-3 \mathrm{p}$ ) the CRC stage IV [23].

This work focuses on the downregulated miRNAs in CRC, miR-145 and miR-365. The miRNA-365 is found situated in the region 13.12 of chromosome 13 and it is present in different stages of regulation according to the type of cancer, being strongly deregulated in the case of glioma, while appearing overregulated in breast cancer [29] or deregulated, based on the type being treated [30]. MiRNA-365 is often down-regulated and involved in regulating cell proliferation, differentiation and apoptosis in numerous cancer cells, like CRC [25,26,31,32]. Bcl2 and Cyclin D1 are some of the target of miRNA-365. Nie et al. [33] demonstrated Cyclin D1 and Bcl-2 are correlated with miRNA-365, and its down-regulation to prognosis in CRC patients. On the other hand, cluster miRNA-143/miRNA-145 is a tumour suppressor usually downregulated in several tumours, being noticeable in the metastatic tumours of CRC patients, but not expressed in epithelial cells [16-18,34]. A significant deregulation of this miRNA has been observed in CRC patients after chemotherapy [35], besides a negative fold change of miRNA-145 in stages II, III and IV CRC [36]. Tanaglu et al. [37] analyzed 16 miRNA expression profiles of 40 patients with recurrent and non-recurrent CRC, finding that the miRNA-145 expression was down-regulated. These data suggest miRNA-145 as a good biomarker for an early detection of this disease [20]. Some of miRNA-145 targets are apoptosis inhibitor5, K-RAS, ERK5 and insulin receptor substrate1 [38]. Exogenous miRNA-145 decreases ERK5 and c-Myc expression, cellular growth and tumour volume in mice [39].

The inhibition of miRNA may use different tools such as miRNA sponges, miRNA masking, antisense oligonucleotides or molecule inhibitors. Anti-miRNA oligonuclotides (AMOs) are being used to suppress dysfunctional miRNAs by steric blocking the target miRNA. AMOs mostly stimulate an RNase-dependent degradation of the target miRNA [40,41]. In contrast to AMOs, miRNA sponges do not degrade the miRNA when they find it, leaving it intact. A miRNA sponge is an mRNA sequence of multiple tandem binding sites to targeted specific miRNAs (MBS), allowing the selective blockade of a complete family of associated miRNAs [42]. The binding specifity of the miRNA towards the end of 3' UTR of its mRNA target is determined by its pairing with the 'seed' sequence [43]. Said sequence contains certain bases which do not pair with the mRNA target, giving place to small loop. Likewise, the sponges containing bulged sites that are mispaired opposite miRNA positions 9-12, show a better and more stable binding efficacy 
than sponges with complete complementarity [44]. This may be caused by an increase in endonucleolytic cleavage activity of AGO2 in the perfect binding of the miRNA that leads to the degradation of the sponge [45].

Usually, sponges contain from four to 10 MBS separated by a few nucleotides each. It has been demonstrated that a greater number of MBS increases the probability of sponge RNA degradation. The efficacy of the sponge also depends on the concentration of sponge RNAs relative to the concentration of the miRNA; therefore, the use of the strong promoters would maximize sponge expression [46]. The main advantage of these miRNA sponges resides in their expression, stable and easily inducible by means of promoters such as U6 or CMV $[47,48]$. The possibility of gene vector-encoded sponges offers new means of controlling miRNAs for therapeutic regulation of gene expression $[49,50]$. Usually, these miRNA sponges are generally designed to connect to reporter genes in order to be well tracked and sorted [44]. Reporter genes are usually placed directly upstream of the MBS, such that the protein expression directly represents sponge RNA expression [46].

We constructed a vector by inserting 7 tandem-arrayed microRNA binding sites to downstream of the CMV (cytomegalovirus) promoter, and used EGFP (enhanced green fluorescent protein) as a 3' UTR reporter gene to analyse the ability of the miRNA sponges to catch the miRNAs 365 and 145. MiRNA sponges must bind to the endogenous cellular miRNA, so the expression of the EGFP gene must be inhibited. This inhibition of EGFP expression levels is therefore proportional to the number of molecules of the endogenous cellular miRNA.

\section{Results}

\subsection{Real-time PCR confirmation of miRNA differential expression}

We worked normalizing with SNORD44 instead of $5 \mathrm{~S}$, due to the first one shows a constitutive expression closer to the miRNAs of interest and a more reliable normalization. The expression of $5 \mathrm{~S}$ is too high (data not shown). Real-time qPCR results revealed that the expression levels of all miRNAs were lower in all of CRC cell lines than in normal colon cell line. Also, the expression levels of miR-145 and miR-365 were similar, being on the HCT116 cell line where minor expression was observed (13.27 \pm 0.64 for miR145 and $11.16 \pm 0.35$ for miR-365) (Fig. 1).

\subsection{Development of a simple and effective miRNA sponge expression system}

Thehsa-miRNA-145-5p and has-miRNA-365-3p sequences were provided by miRNABase database (Fig. 2A). The hsa-miRNA-145-5p and has-miRNA-365-3p sponges were designed to the materials and methods described above (Fig. 2B), synthesized (IDT, Coralville, IO, USA) and subcloned into pEGFP-C1 vector (Clontech) (pC1-EGFP-miR-145; pC1-EGFP-miR-365) (Fig.2 C). 


\subsection{Potential miRNA sponges suppress reporter gene activity}

Flow cytometry analysis revealed that all the cell lines transfected with the pC1-EGFPmiR-145; pC1-EGFP-miR-365 showed decrease of the EGFP expression levels. However, this decline only was significant on the CCD-18Co cell lines (41.9\% and 46.1\%, for miR-145 and miR-365 respectively), while CRC cell lines represented a minimal decline: $21.8 \%$ RKO, 13.7\% T-84 and 9.3\% HTC-116, for miR-145, and 12.4\% RKO, 4.3\% T-84 and 4.6\% HTC-116, for miR-145 (Fig. 3A). These results were corroborated with the decrease in fluorescence observed by fluorescence microscopy (Fig. 3B).

\section{Discussion}

$\mathrm{CRC}$ is one of the most common cancers, with a high risk of metastasis, the main cause of mortality. For this reason, it is necessary to find more effective treatments. Specific patterns of deregulated microRNAs (miRNA) have been associated to diagnosis, prognosis and therapeutic response of tumours, so that they could be used as biomarkers and as a new therapeutic strategy [3,14]. MiRNA-365 and miRNA-145 express lower levels in CRC tumour tissues than in healthy tissues. MiRNA-365 acts as tumour suppressor and that is why its downregulation in CRC leads to a faster tumour proliferation and of worse prognosis, ending in metastasis [51]. MiRNA-145 is related to the inhibition of metastasis and tumour invasion, and for that reason, its downregulation in CRC decontrols these processes [38].

We studied the expression level of these miRNA in the human CRC cells, RKO, T84, SW480, HCT-15, CACO2, HT-29 and HCT-116, and CCD-18co human colon normal cells. At the beginning of the study, we analyzed two housekeeping genes, SNORD44 and 5S. The levels of expression of 5S were higher than those of miRNAs of interest, whereas those of SNORD44 were closer to miR-16, miR-143, miR-145 and miR-365. This fact, together with the frequent use of SNORD44 in comercial kits, such as PerfeCTaR microRNA Assays of Quanta, and that Appaiah et al [52] demonstrated the misuse of 5s for normalization as it showed much variability in several samples, led us to our decision of using SNORD44 to normalize data in the process of quantification of miRNAs. Real-time qPCR results revealed that the expression levels of both miRNAs were lower in all of CRC cell lines than in normal colon cell line. Similar results were obtained by Nie et al [33], whose expression analysis of miR-365 by qPCR was also decreased in the CRC cell lines, HT29, LoVo, SW480 and SW620, compared with normal colon mucosa tissues. Wan et al [53] also corroborated by PCR that the expression levels of miR145 were significantly suppressed in the CRC cell lines, HT-29 and HCT116 cells. In order to make the assays with sponges we chose $3 \mathrm{CRC}$ cell lines: the two lines with higher expression of both miRNAs (RKO and T84) and the line with lower expression (HCT116). As control we used the non-tumour line CCD18-Co. 
Sponges are mRNA molecules able to abduct miRNA molecules thanks to their sequence of multiple tandem binding sites (MBS) [42], allowing to inhibit the expression of diverse families of miRNAs [54]. The use of some sponges have shown an improvement in various diseases: against miR$23 \mathrm{~b}$, reduces tumour malignancy in glioblastoma [55]; against miR-181a, restores chemosensitivity to imatinib mesylate in leukemia [56]; and against miR-221, attenuates neointimal hyperplasia and improves blood flows in vein grafts [57]. The sponge against knockdown of long non-coding RNA called FBXL19-AS1, which is up-regulated in metastatic CRC, inhibits migration, proliferation, tumour growth, invasion and metastasis in vivo [58]. The TUSC7, another long non-coding RNA potential tumour suppressor, could inhibit CRC cell proliferation by completely sponging miR-211-3p [59].

Based on the capability of sponges to abduct specific miRNAs and induce post-transcriptional gene silencing in its target through binding to 3'UTR mRNA[4], and also that the expression profiles of miRNAs are characterised by differing between normal and tumoural tissues[14], we propose to use them to express in a specific way a certain endogenous gene in CRC cell lines, which have a downregulated target miRNA, without affecting the healthy cells, with higher levels of said miRNA.

In this work, two sponges have been designed: one for miRNA-365 and another for miRNA-145, each one of them formed by 7 specific multiple tandem binding sites (MBS). Although the sponge activity is proportional to the number MBS, sponges are usually designed to include 4-12 MBS, because saturation could occur and increase the risk of RNA degradation and genetic recombination increases [44,60]. Kluiver et al [61] designed several sponges against miR19 with different number of MBS, verifying that the one with 6 MBS reaches the maximum inhibition of miR-19, without showing an increase of this inhibition efficiency with a greater number of MBS, finding that the sponge with 12 MBS was less effective. In this research, we did not analyze the correlation between MBS and miRNA inhibition effectiveness, but we show that 7 MBS were sufficient to strongly inhibit miR-145 and miR365 in CCD18Co cells, therefore we do not exclude the possibility that a higher number of MBS may lead to increase this inhibition efficiency. The MBS contain 4 nucleotide mismatches in the middle positions, giving place to small loop. Ebert et al [44] found that sponges with small loop had better and more stable binding efficacy than sponges with complete complementarity. Also, sponges which are perfectly base-paired are vulnerable to Ago2-mediated endonucleolytic cleavage, more easily than sponges with imperfect binding [45]. Besides, MBS were separated by a "spacer", a short 4 nucleotide sequence modification, to optimize the binding of miRNA to every possible MBS and reduce the chance of formation of RNA secondary structure [60].

In order to determine the capability to inhibit the expression of endogenous genes, it was chosen as gene reporter the enhanced green fluorescence protein (EGFP) gen, expressed downstream of the CMV (cytomegalovirus) promoter in the pEGFP-C1 vector (Clontech). Both sponges were subcloned in this plasmid in the 3'UTR of the EGFP (pC1-EGFP-145 and pC1-EGFP-365) and transfected in the 
cell lines. Flow cytometry analysis revealed a decrease of the EGFP expression levels in the cell lines transfected with both sponges with respect to the lines transfected with the pC1-EGFP. This decline was higher on the CCD-18Co cell lines while CRC cell lines presented a minimal decline. In addition to this, this decrease in the EGFP expression was in concordance with the values obtained in the study of qPCR, being inversely proportional to the levels of expression of miRNA-365 and miRNA-145. CCD-18Co cells exposed the highest levels of expression for both miRNAs, and exposed the lowest inhibition to the EGFP expression when transfected by pC1-EGFP-145 and pC1-EGFP-365. Regarding the CRC cell lines, lines RKO and T84, whose levels of expression for both miRNAs were the highest, showed a bigger decrease of the expression of EGFP; however, HCT116, with lower expression of both miRNAs, showed a higher decrease. These results were corroborated by fluorescent microscopy, displaying a decrease in fluorescence in the lines transfected with pC1-EGFP-145 and pC1-EGFP-365, versus the ones transfected with pC1-EGFP, and being that decrease higher in the non-tumour line than in the CRC cell lines. Kluiver [61] subcloned sponges against miRNA-19, one with MBS with perfect complementarity and another with MBS with imperfect complementarity, in the 3'UTR of the GFP. Both sponges were transfected in WEHI-231 cells, murine B-cell lymphoma cells with high expression levels of miRNA-19. In both cases, a decrease in the GFP expression was observed by flow cytometer, being this decrease higher in the sponges forming protuberances than in those which had a perfect complementarity.

Our results show the proper functioning of the vector system, pC1-EGFP-145 and pC1-EGFP365 , to carry in a specific way the expression of genes to CRC genes without affecting healthy cells, preventing damage to healthy tissues.

\section{Conclusion}

Our results show the proper functioning of the vector system, pC1-EGFP-145 and pC1EGFP-365, to carry in a specific way the expression of genes to CRC cells without affecting healthy cells, preventing damage to healthy tissues.

\section{Materials and methods}

\subsection{Cell Culture}

The human colon cancer cells, RKO, T84, SW480, HCT-15, CACO2, HT-29 and HCT116, and CCD- 18co human colon normal cells, were provided by the Instrumentation Service Center of the University of Granada, Granada, Spain. All cell types were grown in Dulbecco's Modified Eagle's Medium (DMEM) (Sigma, St. Louis, MO, USA), supplemented with 10\% fetal bovine serum (FBS) and 1\% streptomycin-penicillin (Sigma), under air containing 5\% $\mathrm{CO}_{2}$ and in an incubator at $37^{\circ} \mathrm{C}$. 


\subsection{RNA Isolation and Real Time-PCR}

Total-RNA from cells was extracted using TRIzol reagent (Invitrogen) according to the manufacturer's instructions. A total of $1 \mu \mathrm{g}$ RNA was used as template for cDNA synthesis. Polymerase (cloned) (Invitrogen) catalyzed the addition of adenosine to the $3^{\prime}$ end of RNA in a sequence-independent fashion. The parameters used were as follows: $37^{\circ} \mathrm{C}$ for $60 \mathrm{~min}$ and $70{ }^{\circ} \mathrm{C}$ for $5 \mathrm{~min}$. Reverse transcription reactions were performed using SuperScript ${ }^{\mathrm{TM}}$ II Reverse Transcriptase (Invitrogen) with universal reverse transcription primer. The reverse transcription thermocycling parameters were as follows: $42{ }^{\circ} \mathrm{C}$ for $80 \mathrm{~min}$ and $85^{\circ} \mathrm{C}$ for $5 \mathrm{~min}$. qPCR was performed using SYBR green supermix (iTaq Universal SYBR Green Supermix (Bio-Rad Laboratories, Hercules, CA). The thermocycling parameters were as follows: $95{ }^{\circ} \mathrm{C}$ for $30 \mathrm{~s}, 95^{\circ}$ for $15 \mathrm{~s}$ and $60^{\circ} \mathrm{C}$ for $60 \mathrm{~s}$. All qRT-PCR assays were performed on an ABI 7900 system (ABI) and $2^{-\Delta \Delta \mathrm{Ct}}$ method was applied for calculation of relative levels of expression. SNORD44 (Small nucleolar RNA 44) was used as the internal reference. Sequences of the miRNA sponge oligonucleotides were obtained from miRbase (www.mirbase.org), and the primers were synthesized according to them (Table 1).

\begin{tabular}{|l|l|}
\hline Mature miRNA & Sequence \\
\hline miR-145-5p & 5' GUCCAGUUUUCCCAGGAAUCCCU 3' \\
\hline miR-365-3p & 5' UAAUGCCCCUAAAAAUCCUUAU 3' \\
\hline SNORD44 & 5' GTCTTAATTAGCTCTAACTGACT 3' \\
\hline 5S & 5'ACCGGGTGCTGTAGGCTTT 3' \\
\hline
\end{tabular}

Table 2: Primers designed for the amplification by qPCR.

\subsection{Sponge design and construction}

The hsa-miRNA-145-5P and has-miRNA-365-3p sequences were provided by miRNABase (miRBase?) database. MicroRNA sponge sequences were chosen based on optimizations previously described by several authors. The features adopted here included: 7 microRNA binding sites(MBS) [46,61,62]; a central bulge with 4 nucleotide mismatches at positions 10 to 13 of the MBS[61,63]; four nucleotide spacer between each MBS[61-63]. For directional cloning, specific restriction site for EcoRI and SacII enzymes were added to the $5^{\prime}$ and 3'-end respectively. Both sponges were synthesized (IDT, Coralville, IO, USA) and subcloned into pEGFP-C1vector (Clontech), which expresses the enhanced green fluorescence protein (EGFP), that was used as reporter gene. Both constructions, miR-145 sponge vector (pEGFP-145) and miR-365 sponge vector (pEGFP-365), were verified by DNA sequencing. 


\subsection{Transfection of cell lines}

One day before transfection, CCD118co, T84, HTC116 and MC38 cell lines were seeded into 24 -well plates $\left(5 \times 10^{4}\right.$ cells per well). Briefly, a transfection mixture was prepared by adding 500ng vector and $1 \mu 1$ Lipofectamine 2000 (Invitrogen). Experiments were performed in four groups: cell lines transfected with pEGFP-145; cell lines transfected with pEGFP-365; cell lines transfected with pEGFP and not transfected cell lines, which were used as controls.

\subsection{Detection by flow cytometry}

We used EGFP as gene reporter to study the ability of miRNA-145 and miRNA-365 to catch their respective miRNA targets. $48 \mathrm{~h}$ after transfection, the cell lines were collected and the changes in the EGFP expression levels were analyzed using the Cellfit program with a FACScan flow cytometer (Becton Dickinson, San Jose, CA, USA).

\subsection{Microscopic Analysis}

EGFP was used as reporter gene in the used constructions as described above. 100nM DAPI (Invitrogen) was used for nuclear staining. EGFP was excited at $488 \mathrm{~nm}$ and DAPI nuclear stain at $364 \mathrm{~nm}$. Fluorescence microscopy analysis was carried out with a Leica DMI6000 microscope (Heidelberg, Germany).

\subsection{Statistical analysis}

Data were present as mean \pm SD of three independent experiments. Statistical significance was evaluated by one-way analysis of variance with Student's t-test using GraphPad Prism 5.0 (GraphPad, CA, USA). The results were considered statistically significant when $\mathrm{p}<0.05$.

\section{Acknowledgments}

This research was funded by FEDER, Plan Nacional de Investigación Científica, Desarrollo e Innovación Tecnológica (I+D+I), Instituto de Salud Carlos III- Fondo de Investigaciones Sanitarias (FIS) through Projects PI11/01862 and PI11/0257.

\section{Conflicts of Interest}

The authors declare no conflict of interest.

\section{Figure and tables legends}

Figure 1. Expression Level of miRNA in cell lines. a) miR-145 level expression; b) miR-365 level expression. 
Figure 2. miRNA sponge Expression System. A) miRNA sequences from miRNABase database. a) hsa-miRNA-145-5p; b) has-miRNA-365-3p. B) Design of miRNA sponges with 7 microRNA binding sites (MBS), a central bulge; four nucleotide spacer between each MBS, and specific restriction site for EcoRI and SacII enzymes for directional clonin. a) miRNA-145-5p sponge; b) miRNA-365-3p sponge; C) Sequence of subcloned into pEGFP-C1 vector.

Figure 3: Analyse of the sponges ability to catch its respective miRNA. A) Flow cytometry analysis of EGFP expression. All cell lines transfected with the pC1-EGFP-miR-145 and pC1EGFP-miR-365 showed decrease of the EGFP expression levels, being higher on the normal cell line while CRC cell lines presented a minimal decline. B) Representative image of detection (20x) of EGFP fluorescence 72 hours after transfection with the pC1-EGFP-miR-145 and pC1-EGFPmiR-365.

Table 1: MiRNA involved in CRC

Table 2: Primers designed for the amplification by qPCR.

\section{References}

1. Bray, F.; Ferlay, J.; Soerjomataram, I.; Siegel, R.L.; Torre, L.A.; Jemal, A. Global cancer statistics 2018: GLOBOCAN estimates of incidence and mortality worldwide for 36 cancers in 185 countries. CA Cancer J Clin 2018, 68, 394-424, doi:10.3322/caac.21492.

2. Siegel, R.L.; Miller, K.D.; Jemal, A. Cancer statistics, 2018. CA Cancer J Clin 2018, 68, 7-30, doi:10.3322/caac.21442.

3. Hernandez, R.; Sanchez-Jimenez, E.; Melguizo, C.; Prados, J.; Rama, A.R. Downregulated microRNAs in the colorectal cancer: diagnostic and therapeutic perspectives. BMB reports 2018, 51, 563-571.

4. Acunzo, M.; Croce, C.M. MicroRNA in Cancer and Cachexia--A Mini-Review. J Infect Dis 2015, 212 Suppl 1, S74-77, doi:10.1093/infdis/jiv197.

5. Kim, Y.; Eom, S.; Park, D.; Kim, H.; Jeoung, D. The Hyaluronic Acid-HDAC3-miRNA Network in Allergic Inflammation. Front Immunol 2015, 6, 210, doi:10.3389/fimmu.2015.00210.

6. Pang, H.L.; Zhao, Q.Q.; Ma, Y.; Song, Y.L.; Min, J.; Lu, J.R.; Li, H.; Zhao, D.Q. Long Noncoding RNA H19 Participates in the Regulation of Adipose-Derived Stem Cells 
Cartilage Differentiation. Stem Cells Int 2019, 2019, 2139814, doi:10.1155/2019/2139814.

7. $\quad$ Fededa, J.P.; Esk, C.; Mierzwa, B.; Stanyte, R.; Yuan, S.; Zheng, H.; Ebnet, K.; Yan, W.; Knoblich, J.A.; Gerlich, D.W. MicroRNA-34/449 controls mitotic spindle orientation during mammalian cortex development. EMBO $J$ 2016, 35, 2386-2398, doi:10.15252/embj.201694056.

8. Sugito, N.; Taniguchi, K.; Kuranaga, Y.; Ohishi, M.; Soga, T.; Ito, Y.; Miyachi, M.; Kikuchi, K.; Hosoi, H.; Akao, Y. Cancer-Specific Energy Metabolism in Rhabdomyosarcoma Cells Is Regulated by MicroRNA. Nucleic Acid Ther 2017, 27, 365377, doi:10.1089/nat.2017.0673.

9. He, M.; Wang, J.; Yin, Z.; Zhao, Y.; Hou, H.; Fan, J.; Li, H.; Wen, Z.; Tang, J.; Wang, Y., et al. MiR-320a induces diabetic nephropathy via inhibiting MafB. Aging (Albany NY) 2019, 11, 3055-3079, doi:10.18632/aging.101962.

10. Femminella, G.D.; Ferrara, N.; Rengo, G. The emerging role of microRNAs in Alzheimer's disease. Front Physiol 2015, 6, 40, doi:10.3389/fphys.2015.00040.

11. Wang, Y.; Zhang, S.; Bao, H.; Mu, S.; Zhang, B.; Ma, H.; Ma, S. MicroRNA-365 promotes lung carcinogenesis by downregulating the USP33/SLIT2/ROBO1 signalling pathway. Cancer Cell Int 2018, 18, 64, doi:10.1186/s12935-018-0563-6.

12. Zhang, H.B.; Sun, L.C.; Ling, L.; Cong, L.H.; Lian, R. miR-143 suppresses the proliferation of NSCLC cells by inhibiting the epidermal growth factor receptor. Exp Ther Med 2016, 12, 1795-1802, doi:10.3892/etm.2016.3555.

13. Mishra, S.; Yadav, T.; Rani, V. Exploring miRNA based approaches in cancer diagnostics and therapeutics. Critical reviews in oncology/hematology 2016, 98, 12-23, doi:10.1016/j.critrevonc.2015.10.003.

14. Liu, G.; Li, B. Role of miRNA in transformation from normal tissue to colorectal adenoma and cancer. Journal of cancer research and therapeutics 2019, 15, 278-285, doi:10.4103/jcrt.JCRT_135_18.

15. Chi, Y.; Zhou, D. MicroRNAs in colorectal carcinoma--from pathogenesis to therapy. Journal of experimental \& clinical cancer research : CR 2016, 35, 43, doi:10.1186/s13046-016-0320-4.

16. Akao, Y.; Nakagawa, Y.; Naoe, T. MicroRNAs 143 and 145 are possible common oncomicroRNAs in human cancers. Oncology reports 2006, 16, 845-850.

17. Takagi, T.; Iio, A.; Nakagawa, Y.; Naoe, T.; Tanigawa, N.; Akao, Y. Decreased expression of microRNA-143 and -145 in human gastric cancers. Oncology 2009, 77, 12 21, doi:10.1159/000218166.

18. Chivukula, R.R.; Shi, G.; Acharya, A.; Mills, E.W.; Zeitels, L.R.; Anandam, J.L.; Abdelnaby, A.A.; Balch, G.C.; Mansour, J.C.; Yopp, A.C., et al. An essential 
mesenchymal function for miR-143/145 in intestinal epithelial regeneration. Cell 2014, 157, 1104-1116, doi:10.1016/j.cell.2014.03.055.

19. You, C.; Liang, H.; Sun, W.; Li, J.; Liu, Y.; Fan, Q.; Zhang, H.; Yue, X.; Chen, X.; Ba, Y. Deregulation of the miR-16-KRAS axis promotes colorectal cancer. Scientific reports 2016, 6, 37459, doi:10.1038/srep37459.

20. Roy, P.; Das, S.; Auddy, R.G.; Mukherjee, A. Engineered andrographolide nanosystems for smart recovery in hepatotoxic conditions. International journal of nanomedicine 2014, 9, 4723-4735, doi:10.2147/IJN.S65262.

21. Imaoka, H.; Toiyama, Y.; Fujikawa, H.; Hiro, J.; Saigusa, S.; Tanaka, K.; Inoue, Y.; Mohri, Y.; Mori, T.; Kato, T., et al. Circulating microRNA-1290 as a novel diagnostic and prognostic biomarker in human colorectal cancer. Annals of oncology : official journal of the European Society for Medical Oncology 2016, 27, 1879-1886, doi:10.1093/annonc/mdw279.

22. Aherne, S.T.; Madden, S.F.; Hughes, D.J.; Pardini, B.; Naccarati, A.; Levy, M.; Vodicka, P.; Neary, P.; Dowling, P.; Clynes, M. Circulating miRNAs miR-34a and miR-150 associated with colorectal cancer progression. BMC cancer 2015, 15, 329, doi:10.1186/s12885-015-1327-5.

23. Kanaan, Z.; Roberts, H.; Eichenberger, M.R.; Billeter, A.; Ocheretner, G.; Pan, J.; Rai, S.N.; Jorden, J.; Williford, A.; Galandiuk, S. A plasma microRNA panel for detection of colorectal adenomas: a step toward more precise screening for colorectal cancer. Annals of surgery 2013, 258, 400-408, doi:10.1097/SLA.0b013e3182a15bcc.

24. Ahmed, F.E. miRNA as markers for the diagnostic screening of colon cancer. Expert review of anticancer therapy 2014, 14, 463-485, doi:10.1586/14737140.2014.869479.

25. Guo, S.L.; Ye, H.; Teng, Y.; Wang, Y.L.; Yang, G.; Li, X.B.; Zhang, C.; Yang, X.; Yang, Z.Z. Akt-p53-miR-365-cyclin D1/cdc25A axis contributes to gastric tumorigenesis induced by PTEN deficiency. Nature communications 2013, 4, 2544, doi:10.1038/ncomms3544.

26. Lea, M.A. Recently identified and potential targets for colon cancer treatment. Future Oncol 2010, 6, 993-1002, doi:10.2217/fon.10.53.

27. Wu, X.; He, X.; Li, S.; Xu, X.; Chen, X.; Zhu, H. Long Non-Coding RNA ucoo2kmd.1 Regulates CD44-Dependent Cell Growth by Competing for miR-211-3p in Colorectal Cancer. PloS one 2016, 11, e0151287, doi:10.1371/journal.pone.0151287.

28. Knudsen, K.N.; Lindebjerg, J.; Nielsen, B.S.; Hansen, T.F.; Sorensen, F.B. MicroRNA$200 \mathrm{~b}$ is downregulated in colon cancer budding cells. PloS one 2017, 12, e0178564, doi:10.1371/journal.pone.0178564. 
29. Zhu, Y.; Zhao, H.; Rao, M.; Xu, S. MicroRNA-365 inhibits proliferation, migration and invasion of glioma by targeting PIK3R3. Oncology reports 2017, 37, 2185-2192, doi:10.3892/or.2017.5458.

30. Han, J.G.; Jiang, Y.D.; Zhang, C.H.; Yang, Y.M.; Pang, D.; Song, Y.N.; Zhang, G.Q. A novel panel of serum miR-21/miR-155/miR-365 as a potential diagnostic biomarker for breast cancer. Annals of surgical treatment and research 2017, 92, 55-66, doi:10.4174/astr.2017.92.2.55.

31. Kang, S.M.; Lee, H.J.; Cho, J.Y. MicroRNA-365 regulates NKX2-1, a key mediator of lung cancer. Cancer letters 2013, 335, 487-494, doi:10.1016/j.canlet.2013.03.006.

32. Zhang, P.; Zheng, C.; Ye, H.; Teng, Y.; Zheng, B.; Yang, X.; Zhang, J. MicroRNA-365 inhibits vascular smooth muscle cell proliferation through targeting cyclin D1. International journal of medical sciences 2014, 11, 765-770, doi:10.7150/ijms.8938.

33. Nie, J.; Liu, L.; Zheng, W.; Chen, L.; Wu, X.; Xu, Y.; Du, X.; Han, W. microRNA-365, down-regulated in colon cancer, inhibits cell cycle progression and promotes apoptosis of colon cancer cells by probably targeting Cyclin D1 and Bcl-2. Carcinogenesis 2012, 33, 220-225, doi:10.1093/carcin/bgr245.

34. Szczyrba, J.; Loprich, E.; Wach, S.; Jung, V.; Unteregger, G.; Barth, S.; Grobholz, R.; Wieland, W.; Stohr, R.; Hartmann, A., et al. The microRNA profile of prostate carcinoma obtained by deep sequencing. Molecular cancer research : MCR 2010, 8, 529-538, doi:10.1158/1541-7786.MCR-09-0443.

35. Drebber, U.; Lay, M.; Wedemeyer, I.; Vallbohmer, D.; Bollschweiler, E.; Brabender, J.; Monig, S.P.; Holscher, A.H.; Dienes, H.P.; Odenthal, M. Altered levels of the oncomicroRNA 21 and the tumor-supressor microRNAs 143 and 145 in advanced rectal cancer indicate successful neoadjuvant chemoradiotherapy. International journal of oncology 2011, 39, 409-415, doi:10.3892/ijo.2011.1036.

36. Xu, X.H.; Wu, X.B.; Wu, S.B.; Liu, H.B.; Chen, R.; Li, Y. Identification of miRNAs differentially expressed in clinical stages of human colorectal carcinoma-an investigation in Guangzhou, China. PloS one 2014, 9, e94060, doi:10.1371/journal.pone.0094060.

37. Tanoglu, A.; Balta, A.Z.; Berber, U.; Ozdemir, Y.; Emirzeoglu, L.; Sayilir, A.; Sucullu, I. microRNA Expression Profile in Patients with Stage II Colorectal Cancer: A Turkish Referral Center Study. Asian Pacific journal of cancer prevention : APJCP 2015, 16, 1851-1855.

38. Wang, W.; Ji, G.; Xiao, X.; Chen, X.; Qin, W.W.; Yang, F.; Li, Y.F.; Fan, L.N.; Xi, W.J.; Huo, Y., et al. Epigenetically regulated miR-145 suppresses colon cancer invasion and metastasis by targeting LASP1. Oncotarget 2016, 7, 68674-68687, doi:10.18632/oncotarget.11919. 
39. Ibrahim, A.F.; Weirauch, U.; Thomas, M.; Grunweller, A.; Hartmann, R.K.; Aigner, A. MicroRNA replacement therapy for miR-145 and miR-33a is efficacious in a model of colon carcinoma. Cancer research 2011, 71, 5214-5224, doi:10.1158/0008-5472.CAN$10-4645$.

40. Lima, J.F.; Cerqueira, L.; Figueiredo, C.; Oliveira, C.; Azevedo, N.F. Anti-miRNA oligonucleotides: A comprehensive guide for design. RNA Biol 2018, 15, 338-352, doi:10.1080/15476286.2018.1445959.

41. Davis, S.; Lollo, B.; Freier, S.; Esau, C. Improved targeting of miRNA with antisense oligonucleotides. Nucleic Acids Res 2006, 34, 2294-2304, doi:10.1093/nar/gkl183.

42. Tang, L.; Chen, H.Y.; Hao, N.B.; Tang, B.; Guo, H.; Yong, X.; Dong, H.; Yang, S.M. microRNA inhibitors: Natural and artificial sequestration of microRNA. Cancer letters 2017, 407, 139-147, doi:10.1016/j.canlet.2017.05.025.

43. Bartel, D.P. MicroRNAs: target recognition and regulatory functions. Cell 2009, 136, 215-233, doi:10.1016/j.cell.2009.01.002.

44. Ebert, M.S.; Neilson, J.R.; Sharp, P.A. MicroRNA sponges: competitive inhibitors of small RNAs in mammalian cells. Nature methods 2007, 4, 721-726, doi:10.1038/nmeth1079.

45. Liu, J.; Carmell, M.A.; Rivas, F.V.; Marsden, C.G.; Thomson, J.M.; Song, J.J.; Hammond, S.M.; Joshua-Tor, L.; Hannon, G.J. Argonaute2 is the catalytic engine of mammalian RNAi. Science 2004, 305, 1437-1441, doi:10.1126/science.1102513.

46. Ebert, M.S.; Sharp, P.A. MicroRNA sponges: progress and possibilities. RNA 2010, 16, 2043-2050, doi:10.1261/rna.2414110.

47. Ebert, M.S.; Sharp, P.A. Emerging roles for natural microRNA sponges. Current biology: CB 2010, 20, R858-861, doi:10.1016/j.cub.2010.08.052.

48. Elcheva, I.; Goswami, S.; Noubissi, F.K.; Spiegelman, V.S. CRD-BP protects the coding region of betaTrCP1 mRNA from miR-183-mediated degradation. Mol Cell 2009, 35, 240-246, doi:10.1016/j.molcel.2009.06.007.

49. Bak, R.O.; Hollensen, A.K.; Mikkelsen, J.G. Managing microRNAs with vector-encoded decoy-type inhibitors. Mol Ther 2013, 21, 1478-1485, doi:10.1038/mt.2013.113.

50. Bak, R.O.; Mikkelsen, J.G. miRNA sponges: soaking up miRNAs for regulation of gene expression. Wiley Interdiscip Rev RNA 2014, 5, 317-333, doi:10.1002/wrna.1213.

51. Bai, J.; Zhang, Z.; Li, X.; Liu, H. MicroRNA-365 inhibits growth, invasion and metastasis of malignant melanoma by targeting NRP1 expression. Cancer biomarkers : section A of Disease markers 2015, 15, 599-608, doi:10.3233/CBM-150500.

52. Appaiah, H.N.; Goswami, C.P.; Mina, L.A.; Badve, S.; Sledge, G.W., Jr.; Liu, Y.; Nakshatri, H. Persistent upregulation of U6:SNORD44 small RNA ratio in the serum of breast cancer patients. Breast cancer research : BCR 2011, 13, R86, doi:10.1186/bcr2943. 
53. Wan, J.; Deng, D.; Wang, X.; Jiang, S.; Cui, R. LINC00491 as a new molecular marker can promote the proliferation, migration and invasion of colon adenocarcinoma cells. OncoTargets and therapy 2019, 12, 6471-6480, doi:10.2147/OTT.S201233.

54. Li, F.; Mahato, R.I. miRNAs as targets for cancer treatment: therapeutics design and delivery. Preface. Advanced drug delivery reviews 2015, 81, v-vi, doi:10.1016/j.addr.2014.11.005.

55. Chen, L.; Zhang, K.; Shi, Z.; Zhang, A.; Jia, Z.; Wang, G.; Pu, P.; Kang, C.; Han, L. A lentivirus-mediated miR-23b sponge diminishes the malignant phenotype of glioma cells in vitro and in vivo. Oncology reports 2014, 31, 1573-1580, doi:10.3892/or.2014.3012.

56. Li, Y.; Kuscu, C.; Banach, A.; Zhang, Q.; Pulkoski-Gross, A.; Kim, D.; Liu, J.; Roth, E.; Li, E.; Shroyer, K.R., et al. miR-181a-5p Inhibits Cancer Cell Migration and Angiogenesis via Downregulation of Matrix Metalloproteinase-14. Cancer research 2015, 75, 2674-2685, doi:10.1158/0008-5472.CAN-14-2875.

57. Wang, X.W.; He, X.J.; Lee, K.C.; Huang, C.; Hu, J.B.; Zhou, R.; Xiang, X.Y.; Feng, B.; Lu, Z.Q. MicroRNA-221 sponge therapy attenuates neointimal hyperplasia and improves blood flows in vein grafts. Int $J$ Cardiol 2016, 208, 79-86, doi:10.1016/j.ijcard.2016.01.006.

58. Shen, B.; Yuan, Y.; Zhang, Y.; Yu, S.; Peng, W.; Huang, X.; Feng, J. Long non-coding RNA FBXL19-AS1 plays oncogenic role in colorectal cancer by sponging miR-203. Biochem Biophys Res Commun 2017, 488, 67-73, doi:10.1016/j.bbrc.2017.05.008.

59. Xu, J.; Zhang, R.; Zhao, J. The Novel Long Noncoding RNA TUSC7 Inhibits Proliferation by Sponging MiR-211 in Colorectal Cancer. Cellular physiology and biochemistry : international journal of experimental cellular physiology, biochemistry, and pharmacology 2017, 41, 635-644, doi:10.1159/000457938.

60. Bernardo, B.C.; Gregorevic, P.; Ritchie, R.H.; McMullen, J.R. Generation of MicroRNA34 Sponges and Tough Decoys for the Heart: Developments and Challenges. Frontiers in pharmacology 2018, 9, 1090, doi:10.3389/fphar.2018.01090.

61. Kluiver, J.; Gibcus, J.H.; Hettinga, C.; Adema, A.; Richter, M.K.; Halsema, N.; SlezakProchazka, I.; Ding, Y.; Kroesen, B.J.; van den Berg, A. Rapid generation of microRNA sponges for microRNA inhibition. PloS one 2012, 7, e29275, doi:10.1371/journal.pone.0029275.

62. Otaegi, G.; Pollock, A.; Sun, T. An Optimized Sponge for microRNA miR-9 Affects Spinal Motor Neuron Development in vivo. Front Neurosci 2011, 5, 146, doi:10.3389/fnins.2011.00146.

63. Kluiver, J.; Slezak-Prochazka, I.; Smigielska-Czepiel, K.; Halsema, N.; Kroesen, B.J.; van den Berg, A. Generation of miRNA sponge constructs. Methods 2012, 58, 113-117, doi:10.1016/j.ymeth.2012.07.019. 
